\title{
Assessment of the social and economic burden of glaucoma in Bulgaria.
}

\author{
Daniela D. Grekova ${ }^{\text {* }}$, Kalina G. Andreevska ${ }^{1}$, Guenka Petrova ${ }^{2}$, Valentin Petkova ${ }^{2}$ \\ ${ }^{1}$ Department of Pharmaceutical Sciences, Medical University of Plovdiv, Bulgaria \\ ${ }^{2}$ Department of Social Pharmacy, Medical University of Sofia, Bulgaria
}

\begin{abstract}
Introduction: Glaucoma commonly refers not to a single disease, but to a group of disorders that have certain common features, in particular cupping and atrophy of the optic nerve head; characteristic visual field loss. Quality of life in glaucoma is affected earlier than previously thought. Aim of this study is to assess the social and economic burdens of glaucoma in Bulgaria.

Materials and methods: The study is a macro observational study of the number of registered and treated patients with glaucoma in Bulgaria, their cost and resulting Disability adjusted life years due to glaucoma.

Results: In 2016 the number of women with glaucoma is 51,321 and the number of male is 27,166. Almost twice as large is the percentage of female suffering from glaucoma-65\%, compared to the percentage of male-35\%. Incidence grows exponentially and in 2014 newly diagnosed were 7975 glaucoma patients, in 2015- 7492 patients, and in 2016-7407 patients. Proportion of newly diagnosed is between $0.08 \%$ and $3.27 \%$ and depends on the age group. Disability adjusted life years for all registered glaucoma patients account for 674,422 and every patient lives on average $10 \mathrm{y}$ in full disability due to visual loss that generates economic losses of $\$ 79000$ per person for life time period.

Conclusions: Glaucoma is a disease that generates economic and social loses to the society and the patients. Bulgarian glaucoma patient's pays half of their pharmacotherapy and bear 10 Disability adjusted life years during their entire life.
\end{abstract}

Keywords: Glaucoma, Cost of pharmacotherapy, Bulgaria, Disability adjusted life years.

Accepted on May 9, 2018

\section{Introduction}

Glaucoma commonly refers not to a single disease, but to a group of disorders that have certain common features, in particular cupping and atrophy of the optic nerve head; characteristic visual field loss; and often, but not invariably, increased intraocular pressure [1].

Of these cardinal signs, visual field loss is diagnostically the most specific, since both cupping and intraocular pressure exhibit physiological variations in a given population [2]. However, visual field loss is a late manifestation of glaucoma, and therefore is not particularly suitable for the early detection of the disease; also, it is not easily investigated reliably in large-scale population screening in developing countries [3].

As the population grows and life expectancy increases, so do the numbers of glaucoma patients and thus the glaucoma burden increases. From population-based studies, $50-80 \%$ of glaucoma patients are not aware of their disease [4]. Quality of life in glaucoma is affected earlier than previously thought. The individual burden of glaucoma impacts many routine daily-life activities. Glaucoma not only impacts glaucoma patients, but also caregivers and all of society. Glaucoma is second only to cataract as a leading cause of global blindness [5], and is the leading cause of irreversible visual loss, largely due to Primary Open-Angle Glaucoma (POAG). In 2002, it was estimated that 161 million individuals worldwide had visual impairment and 37 million were blind. Glaucoma accounted for $12.3 \%$ of global blindness, while cataract accounted for $47.8 \%$. Visual impairment from glaucoma weighs a heavier burden in the least developed regions, and affects adults more than children and women more than men [5]. By the year 2020 this number is predicted to increase to 79.6 million. Globally, glaucoma is a significant cause of vision loss [6]. The prevalence of POAG is expected to increase by an estimated $28 \%$ per decade.

Late interactions with an ophthalmologist and the public's imperfect understanding of the disease contribute to high rates of inadequate control. Uncontrolled glaucoma is therefore a major public health issue [7]. Because of low public awareness and the relatively asymptomatic nature of POAG, delayed presentation is unfortunately commonplace. Many patients diagnosed with glaucoma are blind because of a lack of health care, non-adherence to prescribed therapy, and inadequate treatment. One factor is a lack of education among the highestrisk patients. 
Since publishing the global burden of disease methodology and creating the Disability Adjusted Life Years (DALY) many studies used this as measure for the evaluation of the burden of diseases on the society [8,9]. There is no information about the burden of glaucoma on the Bulgarian society that provokes our study.

The purpose of this study is to assess the social and economic burdens of glaucoma in Bulgaria

The point of view is that of the payer and the patient.

\section{Materials and Methods}

The study is a macro observational study of the number of registered and treated patients with glaucoma in Bulgaria, their cost and resulting DALY due to glaucoma.

\section{Incidence prevalence and cost analysis}

From the National Health Insurance Fund (NHIF) was collected information about the number of registered glaucoma patients for the period 2014-2016. The information was separated as incidence and prevalence, per regions, number and value of reimbursed medicines for glaucoma therapy during 2013-2017 [10].

From the National Social Security Institute was collected information about the yearly registered patients with blindness [11].

\section{DALYs analysis}

Social burden was evaluated through calculation of the disability adjusted life years.

DALY is a single measure for calculating the social burden of illness, mortality and risk factors, and includes lifetimes lost by premature death and years of lower health status.

DALY=Year Life Lost (YLL)+Years Lost due to Disability (YLD) for a given cause (C), Age (A), gender (S) and year (T).

Demographic distribution about age and gender of population was taken from the National Statistical Institute [12]. Life expectancy was considered as 82.2 y for female and 80 for male. Disability weigh was taken from the Global Burden of Diseases and is estimated at 0.7 [13]. Number of years in blindness was taken from the literature as a constant variably of $12.3 \mathrm{y}$ on average [14].

\section{Results}

\section{Incidence, prevalence and cost analysis}

In 2016 the number of women with glaucoma is 51,321 and the number of male is 27,166 . Significantly, almost twice as large is the percentage of female suffering from glaucoma- $65 \%$, compared to the percentage of male- $35 \%$.

In 2016 the city of Sofia as capital has the highest number of registered cases of glaucoma-14,032 patients. Plovdiv ranked second with 7,637 patients, followed by other major citiesStara Zagora-6 501 patients, Varna-5,105 patients, Veliko Tarnovo-3,846 patients and Burgas-3,442 patients. The smallest group of glaucoma patients is registered in Razgrad (Figure 1).

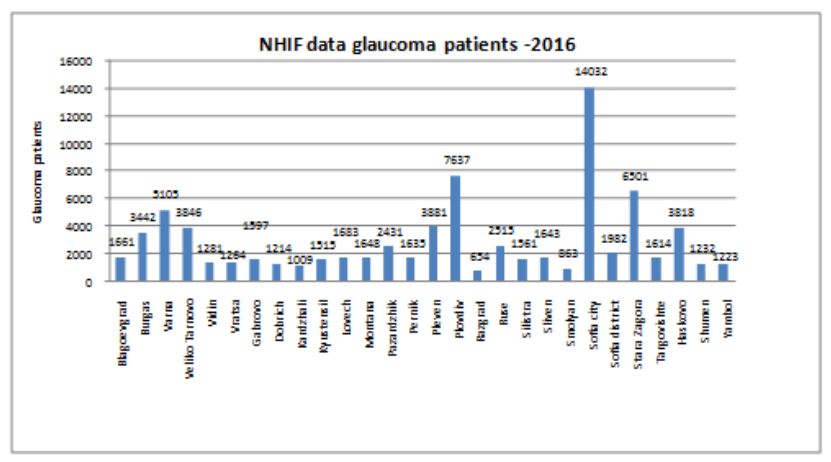

Figure 1. Number of glaucoma patients per regions in 2016.

Comparison of the number of patients with the number of citizens provides information about the proportion of affected people per regions. As a relative share from the total number of population in the regions the highest percentage of glaucoma patients leaves in the city Stara Zagora-2.01\% followed by Haskovo with $1.62 \%$, Veliko Tarnovo- $1.57 \%$ and Pleven-1.54\% as the least is in Blagoevgrad- $0.53 \%$ (Figure 2).

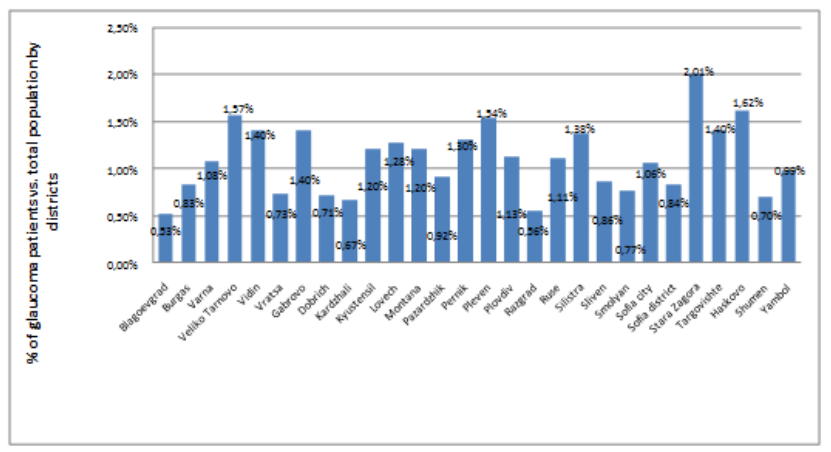

Figure 2. Proportion of glaucoma patients per regions in 2016.

As the disease that progresses with ages the highest is the prevalence in age group above $60-82 \%(n=63,960)$. In age group $40-60$ y relative share is $16 \%(\mathrm{n}=12,842$ patients), and $2 \%$ between $20-40$ y ( $n=1,528$ patients).

Incidence grows exponentially and in 2014 newly diagnosed were 7975 glaucoma patients, in 2015-7492 patients, and in 2016-7407 patients. Proportion of newly diagnosed is $0.08 \%$ in age group 20-40; in age group $40-60 \mathrm{y}$ it is $0.64 \%$ and in the age group over 60 years the prevalence is $3.27 \%$ (Figure 3 ).

In 2016 for 78,487 patients were dispensed 86,053 packages of anti-glaucoma medicines indicating that one patient receive more than one medicinal product, depending on the severity of glaucoma (Figure 4).

Reimbursed cost for anti-glaucoma medicines is decreasing after 2015 probably due to the pricing policy of negotiating discounts with producers and biannual price revision. It should 
be noted that anti-glaucoma medicines are reimbursed $50 \%$ and this may be an economic burden for socially vulnerable old peoples (Table 1).

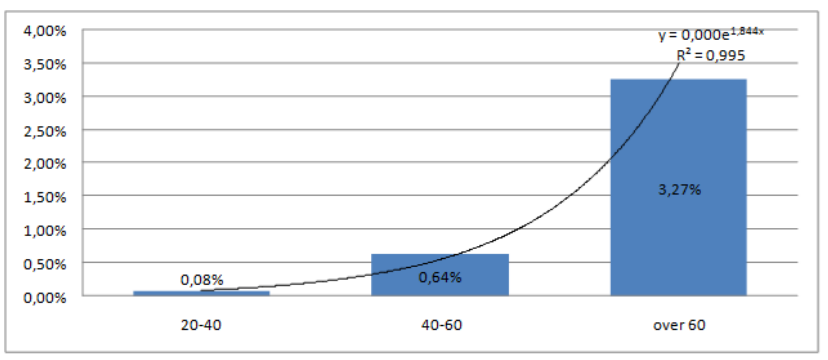

Figure 3. Incidence per age groups in 2016.

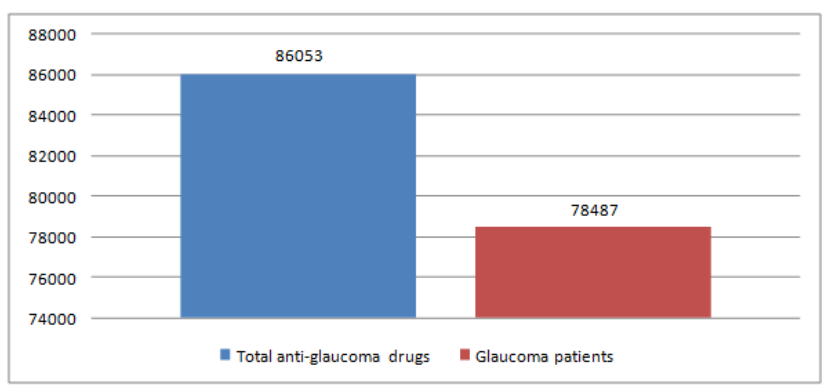

Figure 4. Total number of packages according to number of patients in 2016.

Table 1. Reimbursed cost for anti-glaucoma medicines.

\begin{tabular}{llllll}
\hline Year & $\mathbf{2 0 1 3}$ & $\mathbf{2 0 1 4}$ & $\mathbf{2 0 1 5}$ & $\mathbf{2 0 1 6}$ & $\mathbf{2 0 1 7}$ \\
\hline $\begin{array}{l}\text { Reimbursed } \\
\text { expenditures }\end{array}$ & $3,469,251$ & $3,44,5987$ & $3,534,228$ & $3,251,255$ & $3,339,223$ \\
\hline
\end{tabular}

\section{DALYs analysis}

According to World Health Organization (WHO) the Disability Weight (DW) is calculated by assessing the severity of a life with a particular injury or disability, taking into account the influence of age, concomitant illness and other factors. For a number of conditions, the severity of disability was calculated on a scale of 0 to 1 by using life quality measurement measures. For blindness and depression the disability weight is considered as 0.7 .

Despite the different ages when glaucoma affects the vision and reasons lying behind we consider that on average every glaucoma person will live during $12.3 \mathrm{y}$ of blindness during his/her life. We also assume that there will be no dead due to glaucoma and therefore the number of life yeast lost will equal zero. After these assumptions we calculated the number of years of life in disability as shown on Table 2 .

Table 2. DALYs calculation.

\begin{tabular}{llll}
\hline Age & $\mathbf{2 0 - 4 0}$ & $\mathbf{4 0 - 6 0}$ & Above 60 \\
\hline L-number of years in blindness & 12.3 & 12.3 & 12.3 \\
\hline DW-Disability Weight & 0.7 & 0.7 & 0.7 \\
\hline
\end{tabular}

\begin{tabular}{lccc}
\hline I-number of cases & 1528 & 12842 & 63960 \\
\hline Total YLD $(\mathrm{L} \times \mathrm{DW} \times \mathrm{I})$ & 13156 & 110570 & 550696 \\
\hline
\end{tabular}

After summarizing the YLD the DALY value is equal to 674,422 for all registered patients with glaucoma in case if there is no dead due to the disease, only 12.3 is the average life period with blindness for every age group and disability weigh is 0.7 .

Every patient lives on average 10 years in full disability due to visual loss. The annual gross domestic product per person in 2016 accounts to $\$ 7900$ that generates economic losses of $\$ 79000$ per person for life time period [15].

\section{Discussion}

Our study shows that glaucoma cost is relatively stable but not all costs are visible and patients pay half of the pharmacotherapy cost. There is no official information about the caregivers cost and hospitalizations due to glaucoma. For example in USA the glaucoma annually commands an estimated $\$ 2.5$ billion of the US health care budget, with $\$ 1.9$ billion in direct medical expenses. Health economists estimate that more than $\$ 1.5$ billion is spent on Social Security benefits, lost income tax revenue, and health care expenditures related to glaucoma $[4,16]$. Authors consider that the costs depend on glaucoma severity. The more severe the disease, the costlier is its treatment, as shown by Traverso et al. across several European countries with medical costs ranging from $€ 455$ for earlier stages to $€ 969$ for more advanced disease. In the Unites States this ranged from $\$ 623$ for suspected glaucoma to \$2511 for advanced cases [17]. We did not have information about the severity of the disease because no such exists at macro level.

The amount of the economic burden differs depending on the country. There are large differences between countries concerning annual costs for glaucoma patients [18]. This is mainly from differences in drug prices, and between currencies, whether generics are available and the costs of eye care providers (ophthalmologists or optometrists) and of diagnostic tests [19]. We found that the pharmacotherapy cost per patient is small and account for nearly 46 Euro yearly for medicinal products, and half of them are reimbursed. This could be explained with the thigh price control on the medicines and availability of many generic alternatives on the market.

Our study shows that the social burden in terms of DALYs is huge and it is probably the most important lost for the society.

The limitation of our study is the lack of data at macro level for many types of cost and we calculated only the pharmacotherapy cost. Further microeconomic analysis should be done to reveal the total health care cost of glaucoma.

\section{Conclusion}

Glaucoma is a disease that generates economic and social loses to the society and the patients. Bulgarian glaucoma patient's 
pays half of their pharmacotherapy and bear 10 DALYs during their entire life.

\section{References}

1. WHO. International statistical classification of diseases and related health problems (10th Rev.), (5th Edn.). France, 2015.

2. Bonomi L, Marchini G, Marraffa M. Vascular risk factors for primary open angle glaucoma. The Egna-Neumarkt study. Ophthalmology 2000; 107: 1287-1293

3. Pascolini D, Etyaale D. Global data on visual impairment in the year 2002. Bull WHO 2004; 82: 844-851.

4. Giangiacomo A, Coleman A. The Epidemiology of glaucoma. Essentials in Ophthalmology book series, Springer, Berlin, 2017.

5. Rein DB, Zhang P, Wirth KE. The economic burden of major adult visual disorders in the United States. Arch Ophthalmol 2006; 124: 1754-1760.

6. Quigley HA, Broman AT. The number of people with glaucoma worldwide in 2010 and 2020. Br J Ophthalmol 2006; 90: 262-267.

7. Thylefors B, Negrel AD. The global impact of glaucoma. Bull WHO 1994; 72: 323-326.

8. Murray CJ. Quantifying the burden of disease: the technical basis for disability adjusted life years. Bull WHO 1994; 72: 429-445.

9. Tachkov K, Kamusheva M, Pencheva V, Mitov K. Evaluation of the social and economic burden of chronic obstructive pulmonary disease (COPD). Biotechnol Biotech Equip 2017; 31: 855-861.

10. National Health Insurance Fund. National Framework Contract 2017.

11. National Social Security Institute. Electronic record for hospital lists. http: //www.nssi.bg/index.php/eservicesbg/ eregister
12. National Statistical Institute. Population, demography and immigration. http: //www.nsi.bg/node/2920

13. WHO. WHO methods and data sources for global burden of disease estimates 2000-2011. Geneva: World Health Organization. Global Health Estimates Technical Paper WHO/HIS/HSI/GHE 2013.

14. Varma R, Lee P, Goldberg I, Kotak S.An Assessment of the Health and Economic Burdens of Glaucoma. Am J Ophthalmol 2011; 152: 515-522.

15. USA. Economic indicators. https: // tradingeconomics.com/bulgaria/gdp-per-capita

16. Boland MV, Quigley HA. Risk factors and open angle glaucoma: classification and application. J Glaucoma 2007; 16: 406-418.

17. Lam BL, Zheng DD, Davila EP. Trends in glaucoma medication expenditure: Medical Expenditure Panel Survey 2001-2006. Arch Ophthalmol 2011; 129: 1345-1350.

18. Lee PP, Matchar DB. Economics of glaucoma care. Glaucoma New- York Elsevier 2013.

19. Resnikoff S, Rein DB, Zhang P, Wirth KE. The economic burden of major adult visual disorders in the United States. Arch Ophthalmol 2006; 124: 1754-1760.

\section{${ }^{*}$ Correspondence to}

Daniela D. Grekova

Department of Pharmaceutical Sciences

Medical University of Plovdiv

Bulgaria 